\title{
Substitution of meat and fish with vegetables or potatoes and risk of myocardial infarction
}

\author{
Anne M. L. Würtz ${ }^{1 *}$, Mette D. Hansen ${ }^{1}$, Anne Tjønneland ${ }^{2}$, Eric B. Rimm ${ }^{3,4}$, Erik B. Schmidt ${ }^{5}$, \\ Kim Overvad $^{1,5}$ and Marianne U. Jakobsen ${ }^{1}$ \\ ${ }^{1}$ Section for Epidemiology, Department of Public Health, Aarbus University, DK-800O Aarbus C, Denmark \\ ${ }^{2}$ Danish Cancer Society Research Center, DK-2100 Copenhagen Ø, Denmark \\ ${ }^{3}$ Department of Medicine, Channing Division of Network Medicine, Brigham and Women's Hospital, Harvard Medical School, \\ Boston, MA 02115, USA \\ ${ }^{4}$ Departments of Epidemiology and Nutrition, Harvard T.H. Chan School of Public Health, Boston, MA O2115, USA \\ ${ }^{5}$ Department of Cardiology, Center for Cardiovascular Research, Aalborg University Hospital, DK-900O Aalborg, Denmark
}

(Submitted 3 February 2016 - Final revision received 2 September 2016 - Accepted 5 September 2016 - First published online 24 October 2016)

\section{Abstract}

Red meat has been suggested to be adversely associated with risk of myocardial infarction (MI), whereas vegetable consumption has been found to be protective. The aim of this study was to investigate substitutions of red meat, poultry and fish with vegetables or potatoes for MI prevention. We followed up 29142 women and 26029 men in the Danish Diet, Cancer and Health study aged 50-64 years with no known history of MI at baseline. Diet was assessed by a validated 192-item FFQ at baseline. Adjusted Cox proportional hazard models were used to calculate hazard ratios (HR) and $95 \%$ CI for MI associated with specified food substitutions of $150 \mathrm{~g} /$ week. During a median follow-up of 13.6 years, we identified 656 female and 1694 male cases. Among women, the HR for MI when replacing red meat with vegetables was 0.94 (95\% CI 0.90, 0.98). Replacing fatty fish with vegetables was associated with a higher risk of MI (HR $1.23 ; 95 \%$ CI $1.05,1.45$ ), whereas an inverse, statistically non-significant association was found for lean fish (HR 0.93; $95 \%$ CI 0.83 , 1.05). Substituting poultry with vegetables was not associated with risk of MI (HR 1.00; $95 \%$ CI 0.90, 1.11). Findings for substitution with potatoes were similar to findings for vegetables. Among men, a similar pattern was observed, but the associations were weak and mostly statistically non-significant. This study suggests that replacing red meat with vegetables or potatoes is associated with a lower risk of MI, whereas replacing fatty fish with vegetables or potatoes is associated with a higher risk of MI.

Key words: Myocardial infarction: Meat: Fish: Vegetables: Potatoes: Cohort studies: Substitution models

Myocardial infarction (MI) remains a leading cause of morbidity and mortality in Western countries, and diet is an important modifiable risk factor. Dietary changes usually imply changing the dietary composition rather than the total energy intake ${ }^{(1)}$. Thus, most observational studies on intake of specific foods and risk of $\mathrm{MI}$ include total energy intake in the statistical models. As foods may be beneficial, neutral or harmful in relation to MI development, the association with MI depends not only on the specific food item of interest but also on other foods in the diet that may be replaced by this food item. Studies on foods should therefore specify these substitutions in the statistical models. This will ensure a clear interpretation of the findings, which can easily be translated into dietary guidelines for the general population $^{(2)}$. A study illustrating the importance of specifying the substitutions was conducted by Bernstein et $a l^{(3)}$ : substitution of one daily serving of fish with dairy products was associated with a higher risk of CHD, whereas substitution of one daily serving of red meat with dairy products was associated with a lower risk of CHD.

In observational studies with no specified food substitutions, red meat consumption, particularly processed red meat, has been associated with a higher risk of $\mathrm{MI}^{(3-6)}$, whereas vegetable consumption has been associated with a lower risk of $\mathrm{MI}^{(7,8)}$. Substituting red meat with vegetables may therefore be beneficial for prevention of MI. In addition to red meat, poultry and fish are other major animal dietary protein sources. An inverse association between fish consumption and MI has been suggested $^{(9-12)}$, whereas no association between poultry consumption and MI has been observed ${ }^{(3,6,13)}$. In a meal context, starchy foods such as potatoes constitute a major component besides protein-rich foods and vegetables, and substitutions with potatoes might therefore also be relevant.

Abbreviations: HR, hazard ratio; MI, myocardial infarction.

* Corresponding author: A. M. L. Würtz, fax +45 8613 1580, email amlw@ph.au.dk 
We conducted a follow-up study to investigate substitutions of red meat, poultry and fish with vegetables or potatoes for MI prevention. As meat processing ${ }^{(4)}$, content of $n-3$ PUFA in fish ${ }^{(14)}$ and bioactive components in different vegetables may be important for the association with $\mathrm{MI}^{(15)}$, we also looked into substitutions between subgroups of these foods.

\section{Methods}

\section{Study design and population}

The Diet, Cancer and Health study was initiated in the period between December 1993 and May 1997 by inviting 79729 women and 80996 men, of whom 57053 consented to participate. All were 50-64 years old, living in the greater Copenhagen and Aarhus areas, born in Denmark and not previously registered in the Danish Cancer Registry. The protocols for the Diet, Cancer and Health study and for the present substudy were approved by the regional ethics committees on human studies in Aarhus and Copenhagen and by the Danish Data Protection Agency, and all participants gave their written informed consent. A detailed description of the Diet, Cancer and Health cohort has been published previously ${ }^{(16)}$.

\section{Exposure assessment}

The habitual diet over the past 12 months was assessed using a 192-item, semi-quantitative FFQ filled in by the study participants before their visit to one of the two study centres in Copenhagen and Aarhus. The FFQ was designed for this study and validated against two $7-\mathrm{d}$ weighed diet records ${ }^{(17,18)}$. It was found to be a useful instrument for categorising individuals according to their intake of nutrients and energy ${ }^{(18)}$. The average consumption of foods and beverages was reported by the participants in twelve response categories ranging from 'never' to 'eight times or more per day'. The FFQ was processed by optical scanning to check for reading errors and missing information. Any uncertainty was clarified with the study participant at the visit, and no missing values were accepted. The daily intake of food items was calculated using FoodCalc ${ }^{(19)}$ and sexspecific portion sizes ${ }^{(20,21)}$. The FFQ covered intakes of single meat and fish items and a few mixed dishes such as stew. In total, thirty-five questions covered intakes of red meat, including both unprocessed and processed red meat. Unprocessed red meat included fresh and minced beef, veal, pork and lamb. Processed red meat included red meat items that had undergone processing such as smoking, salting or curing. This included various kinds of sausage, salami, smoked or cooked ham, other cold cuts, bacon and liver pate. A total of four questions covered intake of poultry and included chicken and turkey, whereas twenty-four questions covered intake of fish and shellfish. Total fish included all unprocessed and processed fish and shellfish and was further subdivided into two groups according to the content of $n$-3 PUFA: fish with a low content of $n-3$ PUFA ( $\leq 1 \mathrm{~g} / 100 \mathrm{~g}$ ) included lean white fish, canned tuna, sardines, shellfish and cod roe, and fish with a high content of $n$-3 PUFA ( $>1 \mathrm{~g} / 100 \mathrm{~g})$ included salmon, trout, herring, fresh and canned mackerel, and lumpfish roe. A total of twenty-five questions covered intake of vegetables, including single vegetable items and a few mixed dishes such as salad and vegetable patties. In addition, the vegetable content from standard recipes such as stew contributed small amounts. Vegetable intake was grouped according to botanical and culinary definitions: fruiting vegetables (cucumber, aubergine, green pepper, red pepper, tomato, zucchini, avocado, green beans, green peas, maize), leafy vegetables (spinach, lettuce), root vegetables (carrot, celeriac, ginger), cabbage (cauliflower, broccoli, red cabbage, white cabbage, kale, Brussels sprouts), mushrooms (champignon, chanterelle), onion (onion, garlic) and stalk vegetables/ sprouts (leek, bean sprouts, chives, asparagus, bamboo shoots, rhubarb). Because of the limited intake of leafy vegetables, mushrooms, onions and stalk vegetables/sprouts, these vegetables were combined into one group named 'other vegetables'. A total of eight questions covered the intake of potatoes, including boiled, baked, roasted, fried and mashed potatoes, and they were combined into one potato group, not including potato chips.

\section{Covariates}

At study entry, the participants filled in a lifestyle questionnaire containing questions on health status, social factors, lifestyle habits and reproductive factors. They provided information on smoking habits, physical activity, length of schooling, medical history, hypertension, hypercholesterolaemia and diabetes mellitus; women provided information on menopausal status and use of hormone replacement therapy as well. The lifestyle questionnaire was checked for reading errors, and all unclear or missing information was clarified with the study participants. Trained laboratory technicians recorded the participants' height, weight and waist circumference. Information on alcohol consumption, total energy intake and intake of other foods was obtained from the FFQ.

\section{Case ascertainment}

The outcome measure was incident non-fatal and fatal MI. Participants registered with a first-time discharge diagnosis of MI or cardiac arrest believed to be caused by an MI (International Classification of Diseases, 8th revision, codes 410410.99 and 427.27, and International Classification of Diseases, 10th revision, codes I21.0-I21.9 and I46.0-I46.9) were identified in the Danish National Patient Register and in the Danish Cause of Death Register using the unique ten-digit civil registration number assigned to all Danish citizens by the Central Population Register. From baseline through 2003, the medical records of potential cases were reviewed, and the cases were validated according to MI criteria set by the American Heart Association and the European Society of Cardiology for use in epidemiological studies ${ }^{(22)}$. From 1 January 2004 through 31 December 2009, participants with an MI diagnosis from a hospital ward were accepted as cases without further validation, as the positive predictive value of these register diagnoses from hospital wards was found to be above $92 \%{ }^{(23)}$. Other potential cases were validated by review of diagnoses and procedure codes in the National Patient Register and the Cause of Death Register. 
Information on vital status and emigration was obtained by linkage with the Danish Civil Registration System.

\section{Exclusions}

According to the intention-to-include criteria, participants diagnosed with cancer but not registered in the Danish Cancer Registry because of processing delay before baseline were subsequently excluded. In addition, we excluded participants with a diagnosis of MI or cardiac arrest before enrolment and participants with missing information on potential confounders.

\section{Statistical methods}

The study participants were followed up from the date of study entry until the date of MI diagnosis, emigration, death, loss to follow-up or 31 December 2009, whichever occurred first. Hazard ratios (HR) and 95\% CI for MI were calculated using Cox proportional hazards regression with age as the underlying time scale. The observation time was calculated as the time between study entry and end of follow-up for each participant. The analyses were carried out separately for women and men.

Baseline characteristics of the study participants are summarised using medians and $80 \%$ central ranges for continuous variables and proportions for categorical variables. To illustrate the intake of meat and fish items according to low and high intake of vegetables and potatoes, we created radar charts presenting the percentage-wise difference in median intake of each meat and fish item among women and men in the first and fifth quintiles of intake of total vegetables and of potatoes relative to the median intake of the particular meat and fish item among the entire study population of each sex. For descriptive purposes, intakes of meat, fish, vegetables and potatoes were energy adjusted using the residual $\operatorname{method}^{(24)}$.

We investigated intakes of $150 \mathrm{~g} /$ week, reflecting a usual serving size of meat or fish, for each food item in unspecified and specified substitution models. The specified substitution was introduced into the statistical models by inclusion of a variable made up by the sum of red meat, poultry, fish, vegetables and potatoes in addition to each of these food groups separately, except for the food group to be replaced. As the total amount of red meat, poultry, fish, vegetables and potatoes was held constant by the sum variable, a $150 \mathrm{~g}$ /week higher intake of one of the food groups introduced separately into the model implied a concomitant $150 \mathrm{~g} /$ week lower intake of the specific food group excluded from the model. This means that we specified substitutions of $150 \mathrm{~g} /$ week. In sensitivity analyses, we also investigated unspecified and specified substitutions in kcal/week.

All associations were investigated with adjustment for total energy intake (kcal/week; continuous) (model 1a) and further adjustment for BMI ( $\mathrm{kg} / \mathrm{m}^{2}$; continuous), waist circumference (cm; continuous), alcohol abstinence (yes, no), alcohol intake (g/d; continuous), smoking status and amount (never, former, current $<15 \mathrm{~g}$ tobacco/d, current $15-25 \mathrm{~g}$ tobacco/d and current $>25 \mathrm{~g}$ tobacco/d), leisure time physical activities (dichotomised $;<$ or $\geq 3.5 \mathrm{~h} /$ week $)$ and duration of schooling $(<8,8-10$, $>10$ years) as a measure of socio-economic status, in addition to menopausal status (premenopausal, postmenopausal, unknown) and use of hormone replacement therapy (never, former, current) among women (model 1b). The continuous variables BMI, waist circumference and alcohol intake were entered using restricted cubic splines with three knots. We further adjusted for the following food and beverage groups: fruits, sweets, soft drinks, lean dairy products, fatty dairy products, potato chips, refined cereals, wholegrain cereals and nuts (all g/week; continuous) (model 2). Linearity of the exposure variables was tested using restricted cubic splines and was found to be appropriate. The proportional hazards assumption was explored using the Schoenfeld residuals and graphical assessment. No major violations of the assumptions were identified. To investigate potential effect modification, we initially carried out analyses separately for participants with and without a baseline history of hypertension (yes, no, don't know), hypercholesterolaemia (yes, no, don't know) and/or diabetes mellitus (yes, no, don't know). No substantial differences were found, and we therefore adjusted for these conditions in an additional model (results not shown).

All analyses were performed in Stata versions 13 and 14 (StataCorp LP).

\section{Results}

In total, 57053 participants (29875 women and 27178 men) consented to participate, corresponding to $35 \%$ of those invited. Among these participants, 567 with a cancer diagnosis before baseline, not initially registered, and 900 with a previous diagnosis of MI were excluded. Furthermore, forty-two participants missing a complete baseline examination and 373 with missing information on potential confounders were excluded. These exclusions left 29142 female and 26029 male participants in the present study.

During a median follow-up time of 13.6 years for women and 13.5 years for men, 656 female and 1694 male cases were identified. Baseline characteristics of cohort members and cases are presented in Table 1. Among both women and men, cases were older, had a slightly higher BMI, a larger waist circumference, were more likely to be alcohol abstainers and had a lower alcohol intake compared with cohort members. Furthermore, cases were more likely to be smokers, had a lower level of physical activity and had a shorter length of schooling compared with cohort members. In addition, a higher proportion among cases than among cohort members had a selfreported diagnosis of hypertension, hypercholesterolaemia or diabetes mellitus at baseline. Among women, cases were more likely to be postmenopausal than cohort members, but no differences were observed with regard to use of hormone replacement therapy. The energy-adjusted intakes of red meat, fish, poultry, vegetables and potatoes are presented in Table 2. Radar charts illustrating the intakes of meat and fish items according to low and high intakes of total vegetables and according to low and high intakes of potatoes are presented in the online Supplementary Fig. S1.

The HR and $95 \%$ CI for the incidence of MI associated with the intake of $150 \mathrm{~g} /$ week of each food item separately are presented in Table 3. Among women, intake of total red meat was associated with a statistically significant higher risk of MI in 
Table 1. Baseline characteristics of the cohort and cases in the Diet, Cancer and Health study (Medians and 10th-90th percentiles (p10-p90) or proportions)

\begin{tabular}{|c|c|c|c|c|c|c|c|c|}
\hline \multirow[b]{3}{*}{ Characteristics } & \multicolumn{4}{|c|}{ Women } & \multicolumn{4}{|c|}{ Men } \\
\hline & \multicolumn{2}{|c|}{ Cohort (n 29 142) } & \multicolumn{2}{|c|}{ Cases ( $n$ 656) } & \multicolumn{2}{|c|}{ Cohort (n 26 029) } & \multicolumn{2}{|c|}{ Cases (n 1694) } \\
\hline & Median & p10-p90 & Median & p10-p90 & Median & p10-p90 & Median & p10-p90 \\
\hline \multicolumn{9}{|l|}{ Physiological and anthropometric } \\
\hline Age (years) & 56 & $51-63$ & 59 & $52-64$ & 55 & $51-63$ & 57 & $51-63$ \\
\hline $\mathrm{BMI}\left(\mathrm{kg} / \mathrm{m}^{2}\right)$ & 25 & $21-31$ & 26 & $21-33$ & 26 & $22-31$ & 27 & 23-32 \\
\hline Waist circumference (cm) & 80 & $69-97$ & 84 & $70-103$ & 95 & $84-109$ & 97 & $86-112$ \\
\hline Postmenopausal (\%)* & \multicolumn{2}{|c|}{59} & \multicolumn{2}{|c|}{70} & \multicolumn{2}{|r|}{ 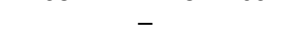 } & \multicolumn{2}{|c|}{-} \\
\hline \multicolumn{9}{|l|}{ Behavioural } \\
\hline Alcohol abstainer (\%) & \multicolumn{2}{|c|}{3} & \multicolumn{2}{|c|}{5} & \multicolumn{2}{|c|}{2} & \multicolumn{2}{|c|}{3} \\
\hline Alcohol intake $(\mathrm{g} / \mathrm{d}) \dagger$ & 10 & $1-35$ & 6 & $1-32$ & 20 & $5-63$ & 19 & $3-64$ \\
\hline Current smoker (\%) & \multicolumn{2}{|c|}{33} & \multicolumn{2}{|c|}{54} & \multicolumn{2}{|c|}{40} & \multicolumn{2}{|c|}{53} \\
\hline$<3.5 \mathrm{~h} /$ week physical activity (\%) & \multicolumn{2}{|c|}{59} & \multicolumn{2}{|c|}{67} & \multicolumn{2}{|c|}{62} & \multicolumn{2}{|c|}{67} \\
\hline$<8$ years of education $(\%)$ & \multicolumn{2}{|c|}{31} & \multicolumn{2}{|c|}{44} & \multicolumn{2}{|c|}{34} & \multicolumn{2}{|c|}{43} \\
\hline Use of hormones (\%)* & \multicolumn{2}{|c|}{30} & \multicolumn{2}{|c|}{30} & \multicolumn{2}{|c|}{-} & \multicolumn{2}{|c|}{-} \\
\hline \multicolumn{9}{|l|}{ Clinical } \\
\hline Diabetes mellitus (\%) & \multicolumn{2}{|c|}{2} & & & & & & \\
\hline Hypertension (\%) & & & & & & & & \\
\hline Hypercholesterolaemia (\%) & & & & & & & & \\
\hline
\end{tabular}

* Among women.

† Among users.

Table 2. Energy-adjusted intakes of red meat, fish, poultry, potatoes and vegetables among cohort and cases in the Diet, Cancer and Health study (Medians and 10th-90th percentiles (p10-p90))

\begin{tabular}{|c|c|c|c|c|c|c|c|c|}
\hline \multirow[b]{3}{*}{ Energy-adjusted dietary intakes (g/week) } & \multicolumn{4}{|c|}{ Women } & \multicolumn{4}{|c|}{ Men } \\
\hline & \multicolumn{2}{|c|}{ Cohort (n 29142) } & \multicolumn{2}{|c|}{ Cases ( $n$ 656) } & \multicolumn{2}{|c|}{ Cohort (n 26 029) } & \multicolumn{2}{|c|}{ Cases (n 1694) } \\
\hline & Median & p10-p90 & Median & p10-p90 & Median & p10-p90 & Median & p10-p90 \\
\hline Total red meat & 582 & $323-883$ & 615 & $374-962$ & 973 & $604-1420$ & 998 & $625-1464$ \\
\hline Unprocessed red meat & 433 & $237-675$ & 443 & $266-705$ & 684 & $401-1060$ & 693 & $396-1059$ \\
\hline Processed red meat & 133 & $46-271$ & 147 & $58-295$ & 260 & $113-481$ & 279 & $123-511$ \\
\hline Fish & 258 & $115-467$ & 259 & $110-469$ & 303 & $133-550$ & 302 & $131-548$ \\
\hline Low $n-3$ PUFA ( $\leq 1 \%)$ & 153 & $69-287$ & 155 & $72-303$ & 178 & 75-339 & 178 & 80-339 \\
\hline High $n-3$ PUFA (>1\%) & 90 & $27-215$ & 87 & $21-206$ & 110 & $34-257$ & 106 & $30-257$ \\
\hline Poultry & 115 & $37-270$ & 107 & $34-259$ & 142 & $48-310$ & 144 & $44-307$ \\
\hline Total vegetables & 1220 & $561-2212$ & 1058 & 461-2052 & 1085 & $490-1973$ & 996 & $430-1923$ \\
\hline Root vegetables & 209 & $47-677$ & 158 & $28-611$ & 119 & $27-454$ & 112 & $18-424$ \\
\hline Cabbage & 112 & $33-250$ & 92 & $27-245$ & 100 & 28-239 & 94 & 24-234 \\
\hline Fruiting vegetables & 457 & 189-920 & 394 & $154-833$ & 410 & $171-842$ & 367 & $151-805$ \\
\hline Potatoes & 774 & $368-1481$ & 796 & $387-1518$ & 1057 & $493-1968$ & 1101 & 492-2022 \\
\hline
\end{tabular}

models adjusted for age, total energy and other established risk factors for MI (model 1b) (HR 1.06; $95 \%$ CI 1.01, 1.10). This association was primarily accounted for by unprocessed red meat (HR 1.08; $95 \%$ CI 1.02, 1.14). Intake of fatty fish (HR 0.82; $95 \%$ CI $0.70,0.95$ ), but not lean fish (HR 1.00; $95 \%$ CI 0.90, $1 \cdot 11$ ), was associated with a statistically significant lower risk of MI. No statistically significant associations were found for poultry, total vegetables or potatoes. Among men, the associations were weak and mostly statistically non-significant. The pattern of associations for red meat and fish were in the same direction as for women, but the direct association between total red meat and MI was accounted for by processed rather than unprocessed red meat.

Table 4 shows the HR and 95\% CI for the incidence of MI per $150 \mathrm{~g} /$ week higher intake of vegetables or potatoes and a concomitant $150 \mathrm{~g} /$ week lower intake of a specified type of red meat, fish or poultry. The results from the age- and energyadjusted models (model 1a) were slightly attenuated when further adjusted for MI risk factors (model 1b). Among women, substituting total vegetables for total red meat was associated with a statistically significant lower risk of MI (HR 0.94; $95 \%$ CI $0 \cdot 90,0 \cdot 98)$. For subgroups of red meat, only substitution of vegetables for unprocessed red meat was statistically significantly associated with MI (HR 0.91; $95 \%$ CI 0.86, 0.98). The HR for substitution of vegetables for processed red meat was 0.99 (95\% CI $0.89,1.09)$. No statistically significant association was found when substituting total vegetables for total fish (HR 1.05 ; $95 \%$ CI $0.96,1.14$ ) or lean fish (HR 0.93; 95\% CI 0.83, $1 \cdot 05)$. However, replacement of fatty fish with vegetables was associated with a higher risk of MI (HR 1.23; $95 \%$ CI 1.05, 1.45). 
Table 3. Myocardial infarction per $150 \mathrm{~g} /$ week higher intake of meat, fish, vegetables or potatoes in the Diet, Cancer and Health study (Hazard ratios (HR) and $95 \%$ confidence intervals)

\begin{tabular}{|c|c|c|c|c|c|c|}
\hline \multirow[b]{3}{*}{ Intakes per $150 \mathrm{~g} /$ week } & \multicolumn{6}{|c|}{ Women (n $29142 / 656)$} \\
\hline & \multicolumn{2}{|c|}{ Model $1 a^{*}$} & \multicolumn{2}{|c|}{ Model 1b† } & \multicolumn{2}{|c|}{ Model $2 \ddagger$} \\
\hline & HR & $95 \% \mathrm{Cl}$ & $\mathrm{HR}$ & $95 \% \mathrm{Cl}$ & $\mathrm{HR}$ & $95 \% \mathrm{Cl}$ \\
\hline Total red meat & $1 \cdot 12$ & $1 \cdot 08,1 \cdot 17$ & 1.06 & $1 \cdot 01,1 \cdot 10$ & 1.06 & $1.01,1 \cdot 11$ \\
\hline Unprocessed red meat & $1 \cdot 14$ & $1.07,1.20$ & 1.08 & $1.02,1.14$ & 1.08 & $1.01,1.15$ \\
\hline Processed red meat & $1 \cdot 23$ & $1 \cdot 13,1.34$ & 1.05 & $0.96,1.16$ & 1.02 & $0.92,1.13$ \\
\hline Total fish & 0.93 & $0.86,1.01$ & 0.94 & $0.87,1.02$ & 0.96 & $0.88,1.03$ \\
\hline Lean fish & 1.01 & $0.91,1.13$ & 1.00 & $0.90,1.11$ & 1.06 & $0.94,1.19$ \\
\hline Fatty fish & $0 \cdot 76$ & $0.66,0.89$ & $0 \cdot 82$ & $0.70,0.95$ & 0.82 & $0.70,0.97$ \\
\hline Poultry & 0.96 & $0.87,1.06$ & 0.97 & $0.88,1.07$ & 1.00 & $0.90,1.10$ \\
\hline Total vegetables & 0.96 & $0.94,0.98$ & 0.99 & $0.97,1.01$ & 1.00 & $0.98,1.02$ \\
\hline \multirow[t]{4}{*}{ Potatoes } & 1.00 & $0.98,1.02$ & 0.98 & $0.96,1.01$ & 0.99 & $0.96,1.01$ \\
\hline & \multicolumn{6}{|c|}{ Men ( $n 26$ 029/1694) } \\
\hline & \multicolumn{2}{|c|}{ Model $1 a^{*}$} & \multicolumn{2}{|c|}{ Model $1 \mathrm{~b} \S$} & \multicolumn{2}{|c|}{ Model $2 \ddagger$} \\
\hline & $\mathrm{HR}$ & $95 \% \mathrm{Cl}$ & $\mathrm{HR}$ & $95 \% \mathrm{Cl}$ & $\mathrm{HR}$ & $95 \% \mathrm{Cl}$ \\
\hline Total red meat & 1.05 & $1.02,1.07$ & 1.02 & $1.00,1.04$ & 1.03 & $1.01,1.06$ \\
\hline Unprocessed red meat & 1.02 & $1.00,1.05$ & 1.01 & $0.98,1.03$ & 1.02 & $0.99,1.05$ \\
\hline Processed red meat & $1 \cdot 14$ & $1.08,1 \cdot 15$ & 1.04 & $1.00,1.08$ & 1.07 & $1.02,1.11$ \\
\hline Total fish & 0.98 & $0.95,1.02$ & 0.99 & $0.96,1.03$ & 1.01 & $0.97,1.06$ \\
\hline Lean fish & 1.01 & $0.95,1.07$ & 1.01 & $0.95,1.07$ & 1.04 & $0.97,1.10$ \\
\hline Fatty fish & 0.93 & $0.86,1.00$ & 0.96 & $0.90,1.04$ & 0.98 & $0.91,1.07$ \\
\hline Poultry & 1.00 & $0.95,1.05$ & 1.03 & $0.98,1.08$ & 1.05 & $1.00,1 \cdot 11$ \\
\hline Total vegetables & 0.97 & $0.96,0.98$ & 1.00 & $0.98,1.01$ & 1.00 & $0.99,1.02$ \\
\hline Potatoes & 1.01 & $1.00,1.02$ & 1.00 & $0.99,1.01$ & 1.01 & $0.99,1.02$ \\
\hline
\end{tabular}

* Adjusted for age and total energy.

† Model 1a was further adjusted for alcohol abstinence, alcohol intake, BMI, waist circumference, smoking status, physical activity, duration of schooling, menopausal status and use of hormone replacement therapy.

¥ Model $1 \mathrm{~b}$ was further adjusted mutually for the investigated food items and for fruits, sweets, soft drinks, lean dairy products, fatty dairy products, potato chips, refined cereals, wholegrain cereals and nuts.

$\S$ Model 1a was further adjusted for alcohol abstinence, alcohol intake, BMI, waist circumference, smoking status, physical activity and duration of schooling.

We found no association with MI when total vegetables replaced poultry (HR 1.00; $95 \%$ CI $0.90,1 \cdot 11$ ). The pattern of replacements of meat or fish items with potatoes was similar to that of meat and fish items replaced with vegetables. The pattern of associations after adjustment for other foods and food groups associated with MI (model 2) was the same as in model 1b. The same pattern of associations was observed with further adjustment for baseline history of hypertension, hypercholesterolaemia and diabetes mellitus (results not shown). Substitutions of meat or fish with vegetable subgroups among women are shown in Fig. 1. Root and fruiting vegetables seemed to be the primary contributors to the findings for total vegetables.

Among men (Table 4), the associations between substitutions of vegetables and potatoes for meat or fish and risk of MI were weak and in general statistically non-significant. These findings did, however, indicate a pattern similar to that of women except for total red meat, where the association seemed to be driven by processed red meat. Substitutions of meat or fish with vegetable subgroups among men are shown in Fig. 2. As for women, the findings for total vegetables seemed primarily to be accounted for by the subgroups of root and fruiting vegetables.

Overall, the findings for unspecified and specified substitutions in $\mathrm{kcal} /$ week were similar to those in $\mathrm{g} /$ week (see online Supplementary Tables S2-S5).

\section{Discussion}

In this long-term, follow-up study, we found a lower risk of MI among women when replacing red meat with vegetables or potatoes. Replacing fatty fish with vegetables or potatoes in contrast was associated with a higher risk of MI. Among men, a similar pattern was observed, but the associations were less likely to be statistically significant.

Individuals usually alter their intake of specific foods primarily by changing the dietary composition rather than by changing the total energy intake unless physical activity or body weight is changed considerably ${ }^{(1)}$. In the specified substitution models, we compared participants who had an identical total energy intake and an identical intake of the total sum of red meat, fish, poultry, vegetables and potatoes, but for whom the intake of each of the individual food groups differed. Inclusion of the sum of red meat, fish, poultry, vegetables and potatoes as well as each of these food groups separately in the statistical models except for the food group to be replaced allowed us to estimate substitutions. This is different from most other studies, as the consumption of these foods has typically been investigated without specifying the replaced food items. In line with previous studies, we also presented risk estimates for the food items one by one without specifying the substitutions. 
Table 4. Myocardial infarction associated with substitution of $150 \mathrm{~g} /$ week of red meat, poultry or fish with vegetables or potatoes in the Diet, Cancer and Health study

(Hazard ratios (HR) and $95 \%$ confidence intervals)

\begin{tabular}{|c|c|c|c|c|c|c|}
\hline \multirow[b]{3}{*}{ Intakes per $150 \mathrm{~g} /$ week } & \multicolumn{6}{|c|}{ Women (n 29 142/656) } \\
\hline & \multicolumn{2}{|c|}{ Model $1 a^{*}$} & \multicolumn{2}{|c|}{ Model 1b† } & \multicolumn{2}{|c|}{ Model $2 \ddagger$} \\
\hline & HR & $95 \% \mathrm{Cl}$ & HR & $95 \% \mathrm{Cl}$ & $\mathrm{HR}$ & $95 \% \mathrm{Cl}$ \\
\hline \multicolumn{7}{|l|}{ Vegetables } \\
\hline Total vegetables for red meat & 0.86 & $0.83,0.90$ & 0.94 & $0.90,0.98$ & 0.94 & $0.89,0.99$ \\
\hline Total vegetables for unprocessed red meat & 0.87 & $0.81,0.93$ & 0.91 & $0.86,0.98$ & 0.92 & $0.86,0.99$ \\
\hline Total vegetables for processed red meat & 0.86 & $0.78,0.95$ & 0.99 & $0.89,1.09$ & 0.98 & $0.88,1.09$ \\
\hline Total vegetables for fish & 1.02 & $0.93,1.10$ & 1.05 & $0.96,1.14$ & 1.04 & $0.96,1.13$ \\
\hline Total vegetables for lean fish & 0.86 & $0.76,0.97$ & 0.93 & $0.83,1.05$ & 0.94 & $0.83,1.06$ \\
\hline Total vegetables for fatty fish & 1.28 & $1.09,1.51$ & $1 \cdot 23$ & $1.05,1.45$ & $1 \cdot 21$ & $1.03,1.42$ \\
\hline Total vegetables for poultry & 0.95 & $0.85,1.05$ & 1.00 & $0.90,1 \cdot 11$ & 0.99 & $0.90,1 \cdot 10$ \\
\hline \multicolumn{7}{|l|}{ Potatoes } \\
\hline Potatoes for red meat & 0.89 & $0.85,0.94$ & 0.93 & $0.88,0.98$ & 0.93 & $0.88,0.98$ \\
\hline Potatoes for unprocessed red meat & 0.90 & $0.84,0.96$ & 0.91 & $0.85,0.97$ & 0.91 & $0.85,0.98$ \\
\hline Potatoes for processed red meat & 0.89 & $0.80,0.98$ & 0.98 & $0.88,1.09$ & 0.97 & $0.87,1.08$ \\
\hline Potatoes for fish & 1.05 & $0.96,1.14$ & 1.04 & $0.96,1.12$ & 1.03 & $0.95,1.12$ \\
\hline Potatoes for lean fish & 0.89 & $0.79,1.00$ & 0.92 & $0.82,1.04$ & 0.93 & $0.83,1.05$ \\
\hline Potatoes for fatty fish & 1.32 & $1 \cdot 12,1.56$ & $1 \cdot 23$ & $1.04,1.44$ & $1 \cdot 20$ & $1.02,1.41$ \\
\hline \multirow[t]{4}{*}{ Potatoes for poultry } & 0.98 & $0.88,1.08$ & 0.99 & $0.90,1 \cdot 10$ & 0.98 & $0.89,1.09$ \\
\hline & \multicolumn{6}{|c|}{ Men ( $n 26$ 029/1694) } \\
\hline & \multicolumn{2}{|c|}{ Model $1 a^{*}$} & \multicolumn{2}{|c|}{ Model 1b§ } & \multicolumn{2}{|c|}{ Model $2 \ddagger$} \\
\hline & HR & $95 \% \mathrm{Cl}$ & $\mathrm{HR}$ & $95 \% \mathrm{Cl}$ & $\mathrm{HR}$ & $95 \% \mathrm{Cl}$ \\
\hline \multicolumn{7}{|l|}{ Vegetables } \\
\hline Total vegetables for red meat & 0.93 & $0.91,0.96$ & 0.98 & $0.96,1.00$ & 0.97 & $0.94,1.00$ \\
\hline Total vegetables for unprocessed red meat & 0.96 & $0.93,0.99$ & 0.99 & $0.96,1.02$ & 0.99 & $0.95,1.02$ \\
\hline Total vegetables for processed red meat & 0.89 & $0.86,0.93$ & 0.96 & $0.92,0.99$ & 0.94 & $0.90,0.98$ \\
\hline Total vegetables for fish & 0.98 & $0.94,1.02$ & 1.00 & $0.96,1.05$ & 0.99 & $0.95,1.03$ \\
\hline Total vegetables for lean fish & 0.93 & $0.87,0.99$ & 0.98 & $0.91,1.04$ & 0.97 & $0.91,1.04$ \\
\hline Total vegetables for fatty fish & 1.05 & $0.96,1.14$ & 1.04 & $0.96,1 \cdot 13$ & 1.02 & $0.94,1.11$ \\
\hline Total vegetables for poultry & 0.94 & $0.89,0.99$ & 0.96 & $0.91,1.01$ & 0.96 & $0.90,1.01$ \\
\hline \multicolumn{7}{|l|}{ Potatoes } \\
\hline Potatoes for red meat & 0.97 & $0.94,0.99$ & 0.98 & $0.96,1.01$ & 0.97 & $0.95,1.00$ \\
\hline Potatoes for unprocessed red meat & 0.99 & $0.96,1.02$ & 1.00 & $0.97,1.02$ & 0.99 & $0.96,1.02$ \\
\hline Potatoes for processed red meat & 0.92 & $0.88,0.96$ & 0.96 & $0.92,1.00$ & 0.94 & $0.91,0.98$ \\
\hline Potatoes for fish & 1.01 & $0.97,1.06$ & 1.01 & $0.97,1.05$ & 1.00 & $0.96,1.04$ \\
\hline Potatoes for lean fish & 0.96 & $0.90,1.03$ & 0.98 & $0.92,1.04$ & 0.98 & $0.92,1.04$ \\
\hline Potatoes for fatty fish & 1.08 & $1 \cdot 00,1 \cdot 18$ & 1.05 & $0.96,1.13$ & 1.02 & $0.94,1.11$ \\
\hline Potatoes for poultry & 0.97 & $0.92,1.02$ & 0.96 & $0.91,1.01$ & 0.96 & $0.91,1.01$ \\
\hline
\end{tabular}

* Adjusted for age and total energy.

† Model 1a was further adjusted for alcohol abstinence, alcohol intake, BMI, waist circumference, smoking status, physical activity, duration of schooling, menopausal status and use of hormone replacement therapy.

‡ Model 1b was further adjusted for fruits, sweets, soft drinks, lean dairy products, fatty dairy products, potato chips, refined cereals, wholegrain cereals and nuts.

$\S$ Model 1a was further adjusted for alcohol abstinence, alcohol intake, BMI, waist circumference, smoking status, physical activity and duration of schooling.

Total energy was included as a covariate in the models, and unspecified substitution models were therefore created $^{(1)}$. Interpretation of these models is not straightforward. The higher intake of the investigated food item is at the expense of other energy-providing food items not included in the model. These food items could be beneficial, neutral or harmful in relation to MI. In other words, the health impact of a specific food item cannot be isolated from that of other foods it replaces, and it is therefore important to specify the substitutions

When investigating substitutions, there are several choices of unit with consequences for the interpretation of the findings. The results presented in this study are based on substitutions of food intakes of $150 \mathrm{~g} /$ week, corresponding to one serving size of meat. However, substitution between protein-rich foods and foods such as vegetables and potatoes might merely be a matter of the relative contribution of each food group to the meal - that is, smaller servings of meat and larger servings of vegetables and potatoes - rather than a complete substitution of one food group with another. When investigating substitutions of similar amounts of foods with different density - for example, meat $v$. vegetables - the volume of the foods substituted differs. This is, however, of minor importance when only small amounts of foods are being substituted. Furthermore, because of differences in energy density of foods, for example, caused by differences in water content, holding total energy constant when investigating substitutions of foods in grams entails a minor unspecified 

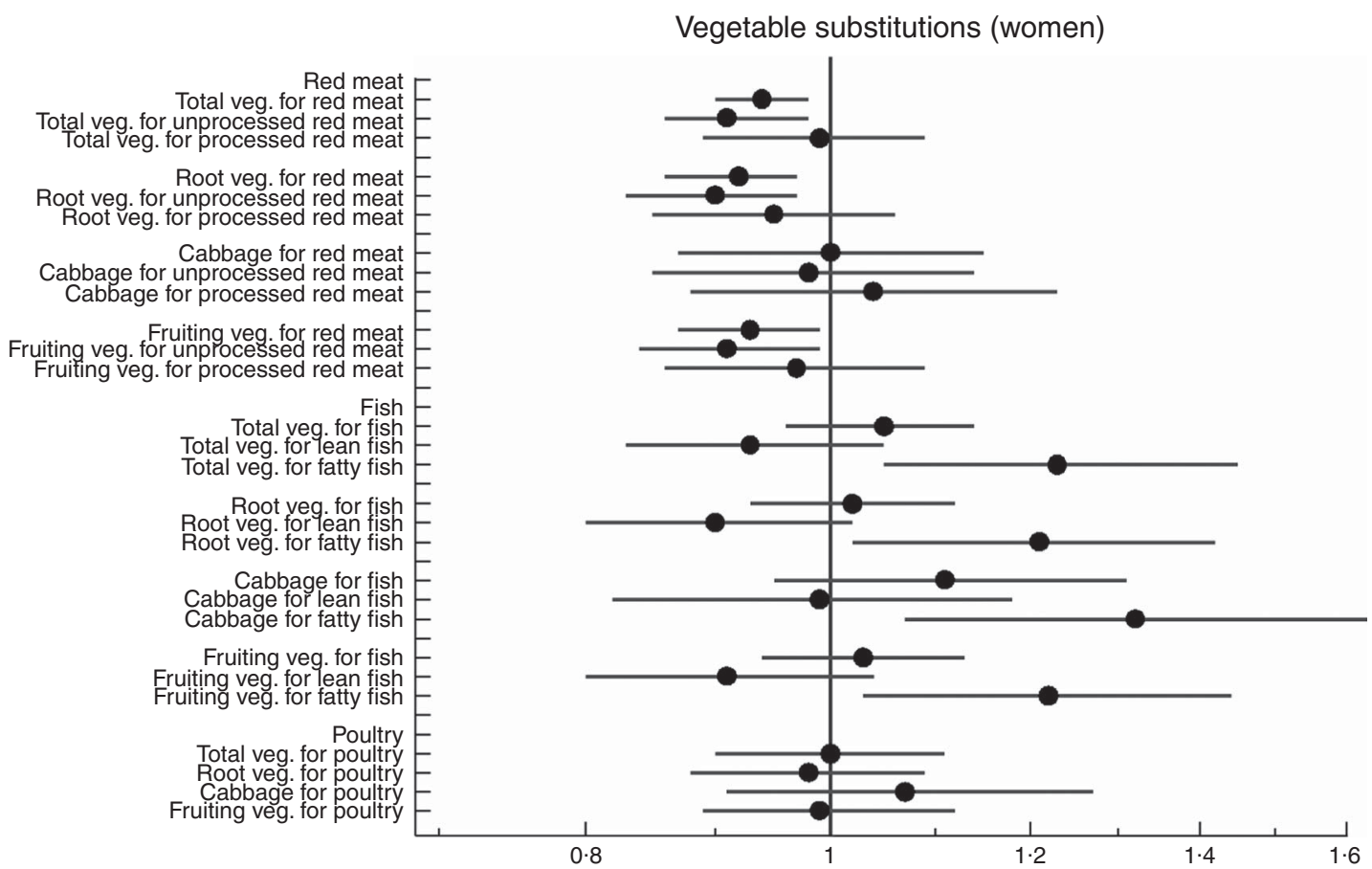

Fig. 1. Hazard ratios and $95 \% \mathrm{Cl}$ for the replacements of red meat, poultry or fish with vegetable subgroups among women adjusted for age, total energy, alcohol abstinence, alcohol intake, BMI, waist circumference, smoking status, physical activity, duration of schooling, menopausal status and use of hormone replacement therapy. Veg., vegetable.

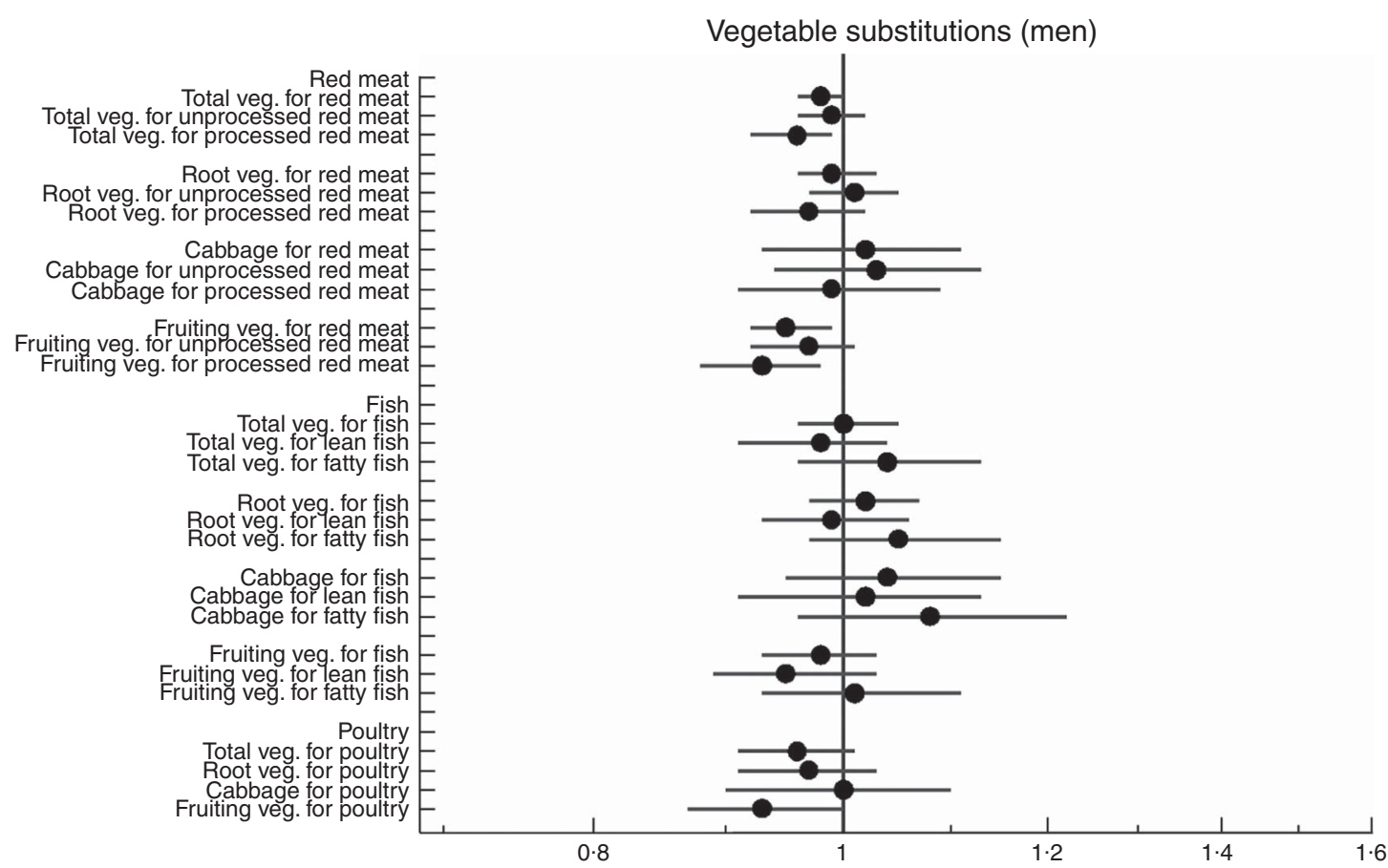

Fig. 2. Hazard ratios and $95 \% \mathrm{Cl}$ for the replacements of red meat, poultry or fish with vegetable subgroups among men adjusted for age, total energy, alcohol abstinence, alcohol intake, BMI, waist circumference, smoking, physical activity and duration of schooling. Veg., vegetable.

residual substitution of energy from other foods. We also investigated substitutions of equal amounts of energy from the different foods. This ensures no residual substitution due to differences in energy density, but the amounts of the two foods substituted differ according to differences in the energy density of the foods investigated. The patterns of results for substitutions in energy/week were overall similar to those in $\mathrm{g} /$ week. A third choice is substitution of servings (volume or weight specific), as applied in the study by Bernstein et $a l^{(3)}$. The same issue with unspecified substitution occurs with varying energy density of 
the substituted foods when using servings. An additional potential cause of differences in energy content between the foods being substituted is the difference in serving sizes, unique for each food item of interest.

Statistically, there are several options for modelling substitutions. In the present study, we included a sum variable that contained all the food groups to be substituted between in addition to each of these food groups separately, except for the food group to be replaced. Thereby, we obtained the HR and corresponding $95 \%$ CI for the substitutions directly in the output from the Cox model. Another method to obtain substitution estimates is to include all food groups to be substituted between in the same model with no sum variable ${ }^{(3,25)}$. From the output, the difference between the $\beta$-coefficients of two food groups is used to estimate the HR for the substitution between these two food groups. The variance of each food group and the covariance of the two food groups are used to calculate the corresponding $95 \%$ CI. These two methods to obtain HR and $95 \%$ CI for substitutions are equivalent, which is evident from the re-parametrisation in the online Supplementary Material S6.

Because of inclusion criteria, participants of higher socioeconomic status were slightly over-represented in the study cohort $^{(16)}$. However, within the cohort, there was substantial exposure variation. The follow-up of participants in the study was nearly complete, which reduces the concern for selection bias. However, censoring due to death from other causes with disease aetiology similar to that of MI may have introduced selection bias, and the true associations may thus have been underestimated. For example, participants who were censored due to death from other atherosclerotic diseases such as stroke most likely also had a higher risk of MI due to similarities in the underlying aetiology.

The dietary information was obtained from self-administered FFQ, which are affected by random measurement error. This generally leads to underestimation of the true association and to loss of statistical power. Furthermore, differential reporting of different foods cannot be excluded, and potential systematic under- and over-reporting could bias the estimates ${ }^{(26,27)}$. The comprehensive information on intake of subtypes of food groups enabled us to investigate subgroup-specific associations. Although FFQ may reflect the habitual eating pattern and we aimed to assess the long-term association between different food choices and development of MI, repeated measures would have been preferred over a single baseline measure. Multiple measures would have allowed us to correct some measurement error, and dietary intake might have changed during follow-up. The use of complete registries for information on diagnosis of $\mathrm{MI}$ in addition to the confirmation and validation of each case of MI minimises the risk of misclassification of the outcome. As the diagnoses of MI were established independently of the dietary information, information bias is unlikely to have affected our results.

The detailed information on potential confounders limits the risk of residual confounding, but confounding from risk factors of MI not taken into consideration cannot be excluded. Adjusting our models for established risk factors of MI weakened the risk estimates, indicating confounding from these risk factors. In the food substitution models not adjusted for other foods (model 1b), the underlying dietary pattern associated with different choices of meat, fish, vegetables or potatoes was allowed to vary accordingly. Thus, the food items substituted may be considered as indicators of the underlying dietary patterns. By contrast, in model 2, further adjusted for selected foods and food groups, only the meat, fish, vegetable and potato items specified in the substitution models and the dietary factors not adjusted for were allowed to vary, thereby restricting the underlying dietary patterns. Thus, in model 2 , the food substitution per se was investigated, but the findings may not easily be applied to a meal context. However, in model $1 \mathrm{~b}$, confounding from other dietary factors cannot be excluded. In addition, the unspecified residual substitution occurring because of differences in energy density of the foods being substituted is restricted by adjustment for other dietary factors. Therefore, the interpretation of findings differs between models with and without adjustment for other foods, even though the overall pattern of associations in the two models was similar.

In the Nurses' Health Study of US female nurses, Bernstein et $a l .{ }^{(3)}$ found no association with CHD when one daily serving of red meat, poultry or fish was replaced with one serving of beans. Likewise, in a study of US women and men, Haring et $a l .{ }^{(28)}$ found no association with CHD when one serving of red meat, processed meat, poultry or fish and seafood was replaced with one serving of legumes. Because of a limited intake of beans and other legumes, we were not able to explore these substitutions specifically in the present study.

Because of differences in method of preparation and contents of specific fats, $\mathrm{Na}$, nitrites and other additives, we hypothesised that replacement of processed red meat with vegetables or potatoes would be more beneficial than replacement of unprocessed red meat ${ }^{(4)}$. This pattern was only recognised among men, and our results did not confirm this hypothesis among women. For fish, we found that replacement of lean fish with vegetables or potatoes was inversely associated with MI among both women and men, but was not statistically significant, whereas the results for fatty fish replaced with these foods suggested direct associations with MI. This could be explained by the higher content in fatty fish of $n$-3 PUFA with their presumed beneficial effects on $\mathrm{CHD}^{(14)}$. Our findings among men were weaker and less likely to be statistically significant than our findings among women. This could be explained by differences in the baseline hazard. As men have a higher baseline risk of MI than women, the associations on a relative scale are expected to be weaker.

In conclusion, replacing red meat with vegetables or potatoes was associated with a lower risk of MI, and replacing fatty fish with vegetables or potatoes was associated with a higher risk of MI. Studies on individual dietary changes over time and subsequent risk of MI would add additional value to elucidate the role of food substitutions in the risk of future MI.

\section{Acknowledgements}

The authors thank the Danish Cancer Society and the staff of the Diet, Cancer and Health study for the collection and administration of data.

This work is part of the project 'Diet and prevention of ischemic heart disease: a translational approach' (DIPI, www.dipi.dk), 
which is supported by the Danish Council for Strategic Research (today, Innovation Fund Denmark) (Contract 0603-00488B). One-third of the fellowship was granted by the Graduate School of Health, Aarhus University. The primary data collection was funded by the Danish Cancer Society. The funding agencies had no role in the study design, analysis or writing of this article.

The authors' contributions are as follows: A. M. L. W., K. O. and M. U. J. contributed to the study design; A. T. and K. O. collected the data; A. M. L. W. performed the statistical analyses; A. M. L. W., M. D. H., A. T., E. B. R., E. B. S., K. O. and M. U. J. were involved in the interpretation of the data and critical revision of the manuscript; A. M. L. W. wrote the manuscript. A. M. L. W. had primary responsibility for the final content. All authors read and approved the final version of the manuscript.

The authors declare that there are no conflicts of interest.

\section{Supplementary material}

For supplementary material/s referred to in this article, please visit http://dx.doi.org/doi:10.1017/S0007114516003500

\section{References}

1. Willett WC, Howe GR \& Kushi LH (1997) Adjustment for total energy intake in epidemiologic studies. Am J Clin Nutr $\mathbf{6 5}$, Suppl., 1220S-1228S; discussion 1229S-1231S.

2. Astrup A, Dyerberg J, Elwood P, et al. (2011) The role of reducing intakes of saturated fat in the prevention of cardiovascular disease: where does the evidence stand in 2010? Am J Clin Nutr 93, 684-688.

3. Bernstein AM, Sun Q, Hu FB, et al. (2010) Major dietary protein sources and risk of coronary heart disease in women. Circulation 122, 876-883.

4. Micha R, Wallace SK \& Mozaffarian D (2010) Red and processed meat consumption and risk of incident coronary heart disease, stroke, and diabetes mellitus: a systematic review and meta-analysis. Circulation 121, 2271-2283.

5. Pan A, Sun Q, Bernstein AM, et al. (2012) Red meat consumption and mortality: results from 2 prospective cohort studies. Arch Intern Med 172, 555-563.

6. Takata Y, Shu XO, Gao YT, et al. (2013) Red meat and poultry intakes and risk of total and cause-specific mortality: results from cohort studies of Chinese adults in Shanghai. PLOS ONE $\mathbf{8}, \mathrm{e} 56963$.

7. Dauchet L, Amouyel P, Hercberg S, et al. (2006) Fruit and vegetable consumption and risk of coronary heart disease: a meta-analysis of cohort studies. J Nutr 136, 2588-2593.

8. Dauchet L, Amouyel P \& Dallongeville J (2009) Fruits, vegetables and coronary heart disease. Nat Rev Cardiol 6, 599-608.

9. Kris-Etherton PM, Harris WS, Appel LJ, et al. (2002) Fish consumption, fish oil, omega-3 fatty acids, and cardiovascular disease. Circulation 106, 2747-2757.

10. Calder PC (2004) n-3 Fatty acids and cardiovascular disease: evidence explained and mechanisms explored. Clin Sci (Lond) 107, 1-11.

11. Whelton SP, He J, Whelton PK, et al. (2004) Meta-analysis of observational studies on fish intake and coronary heart disease. Am J Cardiol 93, 1119-1123.
12. He K, Song Y, Daviglus ML, et al. (2004) Accumulated evidence on fish consumption and coronary heart disease mortality: a meta-analysis of cohort studies. Circulation 109, 2705-2711.

13. Sinha R, Cross AJ, Graubard BI, et al. (2009) Meat intake and mortality: a prospective study of over half a million people. Arch Intern Med 169, 562-571.

14. De Caterina R (2011) n-3 Fatty acids in cardiovascular disease. $N$ Engl J Med 364, 2439-2450.

15. Slavin JL \& Lloyd B (2012) Health benefits of fruits and vegetables. Adv Nutr 3, 506-516.

16. Tjonneland A, Olsen A, Boll K, et al. (2007) Study design, exposure variables, and socioeconomic determinants of participation in Diet, Cancer and Health: a population-based prospective cohort study of 57,053 men and women in Denmark. Scand J Public Health 35, 432-441.

17. Overvad K, Tjonneland A, Haraldsdottir J, et al. (1991) Development of a semiquantitative food frequency questionnaire to assess food, energy and nutrient intake in Denmark. Int J Epidemiol 20, 900-905.

18. Tjonneland A, Overvad K, Haraldsdottir J, et al. (1991) Validation of a semiquantitative food frequency questionnaire developed in Denmark. Int J Epidemiol 20, 906-912.

19. Lauritsen J (1999) FoodCalc. http://www.ibt.ku.dk/jesper/ foodcalc/ (accessed January 2016).

20. Haraldsdottir J, Tjonneland A \& Overvad K (1994) Validity of individual portion size estimates in a food frequency questionnaire. Int J Epidemiol 23, 786-796.

21. Tjonneland A, Haraldsdottir J, Overvad K, et al. (1992) Influence of individually estimated portion size data on the validity of a semiquantitative food frequency questionnaire. Int $J$ Epidemiol 21, 770-777.

22. Luepker RV, Apple FS, Christenson RH, et al. (2003) Case definitions for acute coronary heart disease in epidemiology and clinical research studies: a statement from the AHA Council on Epidemiology and Prevention; AHA Statistics Committee; World Heart Federation Council on Epidemiology and Prevention; the European Society of Cardiology Working Group on Epidemiology and Prevention; Centers for Disease Control and Prevention; and the National Heart, Lung, and Blood Institute. Circulation 108, 2543-2549.

23. Joensen AM, Jensen MK, Overvad K, et al. (2009) Predictive values of acute coronary syndrome discharge diagnoses differed in the Danish National Patient Registry. J Clin Epidemiol 62, 188-194.

24. Willett W \& Stampfer MJ (1986) Total energy intake: implications for epidemiologic analyses. Am J Epidemiol 124, $17-27$.

25. Muraki I, Imamura F, Manson JE, et al. (2013) Fruit consumption and risk of type 2 diabetes: results from three prospective longitudinal cohort studies. BMJ 347, f5001.

26. Heitmann BL \& Lissner L (2005) Can adverse effects of dietary fat intake be overestimated as a consequence of dietary fat underreporting? Public Health Nutr 8, 1322-1327.

27. Heitmann BL \& Lissner L (1995) Dietary underreporting by obese individuals - is it specific or non-specific? BMJ $\mathbf{3 1 1}$, 986-989.

28. Haring B, Gronroos N, Nettleton JA, et al. (2014) Dietary protein intake and coronary heart disease in a large community based cohort: results from the Atherosclerosis Risk in Communities (ARIC) study [corrected]. PLOS ONE 9, e109552. 